\title{
Improvement of Dysphagia in a Chronic Brain Injury Patient with Temporomandibular Joint Dysfunction: A Case Report
}

\author{
Kyu Wan Kwak, M.D. ${ }^{1}$, Hyo Jeong Lee, M.D. ${ }^{2}$, Yoon Ghil Park, M.D., Ph.D. ${ }^{1}$ \\ ${ }^{1}$ Department of Rehabilitation Medicine, Gangnam Severance Hospital, Rehabilitation Institute of Neuromuscular \\ Disease, Yonsei University College of Medicine, Seoul, ${ }^{2}$ Department of Rehabilitation Medicine, Bundang Jesaeng \\ Hospital, Seongnam, Korea
}

\begin{abstract}
Acquired brain injury patients who present dysphagia associated with temporomandibular joint dysfunction (TMJD) are more common than expected. TMJD is associated with restriction of mouth opening and jaw movements, thereby interfering with the oral phase of the swallowing process. An 18-year-old female with traumatic brain injury mainly complained of dysphagia when first admitted. After nearly one year of conventional dysphagia therapy, the patient could barely eat a minced diet. The videofluoroscopic swallowing study showed that a soft and bite-sized diet was tolerable to the patient, but the restricted mouth opening and mandibular movement imposed a major limitation of the dietary build-up. After examining the imaging studies, the patient was diagnosed with TMJD attributed to muscle lesion and misaligned stomatognathic system. The patient subsequently underwent a stomatognathic alignment and TMJ strengthening exercise program over a span of two weeks, after which the therapeutic effects were evaluated with the craniomandibular index (CMI) and Mann Assessment of Swallowing Ability (MASA) parameters, before and after the exercise program. The results showed improvements in the neck mobility and orofacial movements, and the patient was able to consume soft and bite-sized diet in the clinical setting. Thus, we propose that active exercise to achieve postural correction and TMJ muscle strengthening are effective treatment options for dysphagia associated with TMJD. (JKDS 2021;11:147-153)
\end{abstract}

Keywords: Acquired brain injury, Temporomandibular joint dysfunction, Stomatognathic alignment exercise

\section{INTRODUCTION}

Traumatic brain injury (TBI) and stroke-related sensorimotor impairment in stomatognathic system including head and neck muscles, masticatory muscles, and temporomandibular joint (TMJ) results in strength loss in lip, tongue, masticatory muscles, restriction of bite force and poor quality of mastication $^{1}$. In specific, temporomandibular joint dysfunction (TMJD) is a collective term, which consists of masticatory muscle disorders and mandibular hypomobility disorders specific to the joint ${ }^{2}$. TMJD is clinically associated with restriction of mouth opening, limitation of the range of motion

Received: April 12 2021, Revised: April 13 2021,

Accepted: May 252021

Corresponding author: Yoon Ghil Park, Department of Rehabilitation Medicine, Gangnam Severance Hospital, Yonsei University, 20 Eonju-ro 63-gil, Gangnam-gu, Seoul 06229, Korea Tel: +82-2-2019-3498, Fax: +82-2-2019-4881

E-mail:DRTLC@yuhs.ac

Copyrights (c) The Korean Dysphagia Society, 2021. 
(ROM) in jaw, pain in joint by palpation, and deviation of the jaw in mouth opening and locking sensation. This is due to the fact that a variety of movements occur at the TMJ: mandibular depression, elevation, lateral deviation, retrusion and protrusion. Each of these movements are performed by a number of muscles working together to perform the movement while keeping the position of the condylar head of the mandible within the mandibular fossa of the temporal bone. These muscles are temporalis, masseter, lateral/medial pterygoid, digastric, stylohyoid, mylohyoid, geniohyoid, platysma, ${ }^{3,4}$. Prevalence of TMJD varies depending on the pathology; however, it is reported that $75 \%$ of the general population had asymptomatic TMJD, and only $3.6 \%-7.5 \%$ of them admit for medical attention ${ }^{5}$.

The purpose of this case report is to evaluate the effect of stomatognathic alignment exercise and TMJ strengthening exercise on a chronic patient with TMJD attributed to TBI and postoperative sequelae, mostly focusing on the assessment of temporomandibular range of motion and mastication quality.

\section{CASE REPORT}

On December 18th, 2017, an 18-year-old female patient history sustained head trauma after pedestrian traffic accident. The patient went to the emergency room of a nearby hospital and initial brain CT image showed Lt. EDH, SDH, so the patient was transferred to neurological surgery department and underwent craniectomy and hematoma removal, duroplasty on the day of arrival. On October 15th, 2018, the patient went through ventriculoperitoneal (VP) shunt operation due to hydrocephalus. On March 22nd, 2019, the patient underwent cranioplasty with 3D printing implant due to extensive skull defect of Lt. frontotemporal area.

After quite some demanding schedule, when the patient was first admitted to tertiary hospital for rehabilitation on July 7th, 2019, the chief complaint included not only quadriplegia and cognitive impairment but also included dysphagia. Oropharyngeal function test and videofluoroscopic swallowing study (VFSS) conducted on September 24th, 2019 revealed an obvious decrease in labial closure and rotatory tongue movement, slow oral transit, reduced pharyngeal propulsion, incomplete elevation and closure of larynx, penetration with large amount of semisolid, and aspiration with large amount of liquid during swallowing. From that point, with the help from the conventional dysphagia therapy, the patient could terminate nasogastric tube feeding and proceed to diet level 4 (pureed food), using the international dysphagia diet standardization initiative (IDDSI) ${ }^{6}$. By the time the patient was admitted to department of rehabilitation at tertiary hospital for the 3rd time on March 5th, 2020, dietary build-up had already been leveled up to diet level 5 (minced and moist food) and no swallowing aspiration events had been observed in the meantime. The follow-up VFSS on March 6th, 2020 showed only marginal improvements: the swallowing of diet level 6 (soft and bite-sized food) was tolerable, yet the swallowing of small liquid $(5 \mathrm{cc}$ ) resulted in during swallowing aspiration. In clinical setting, soft and bite-sized diet with the exception of liquid was tolerable to the patient. Hence, therapeutic goal was set to build up to diet level 6 (soft and bite-sized food) that require more efficient mastication. However, there was a major obstacle to this: restricted mouth opening and mandibular movement.

The patient had cognitive impairment, getting 5 points in a mini-mental state examination (MMSE), but she could follow simple commands in relation to basic daily needs. When spoon-feeding was provided to the patient, she could barely open her mouth with the more severe degree of restriction toward right. Since brain injury is one of the important causes for TMJD regardless of ischemia or hemorrhage, the patient was physically examined with suspected TMJD $^{7}$. Initial assessment on March 11th, 2020 showed that maximal mouth opening (MMO) measured from incisor to incisor with caliper was $10 \mathrm{~mm}$, while passive stretch opening was $13 \mathrm{~mm}$ with hard end-feel. Given that normal interincisal opening is 
approximately $35-45 \mathrm{~mm}^{8}$, the patient showed a severe degree of reduced mouth opening. Also, intermittent opening click was heard on Rt. in the early phase of mouth opening, possibly suggesting disc displacement with reduction at Rt. TMJ. The patient also complained of Lt. TMJ pain, showing tenderness with palpation of both masseter muscles. On imaging studies, TMJ tomogram showed rotational restriction of Lt. mandibular condyle; in the meantime, Panoramic dental X-ray showed severe narrowing of both TMJ spaces.(Fig. 1) However, facial bone CT taken for precise evaluation of TMJ showed no adhesion or ankylosis.(Fig. 2) This led to the following conclusion: the patient's restricted mouth opening could be more likely due to Lt. temporalis muscle lesion and contracture related TMJD as a postoperative complication of cranioplasty with 3D printing implant.

Based on the studies' findings, in addition to traditional dysphagia therapies that mainly focus on the pharyngeal and esophageal phase of swallowing, we planned to implement stomatognathic alignment ${ }^{9,10}$ and TMJ strengthening exercise program - aimed at maximization of the efficiency of the oral phase to the patient. A core part of the stomatognathic alignment exercise program was postural correction exercise: passive and active range of motion exercises of the TMJ and neck were applied to the patient in sitting position with the thoracic and lumbar spines being supported by a back support. For TMJ strengthening, the therapist placed a thumb under the patient's chin and pushed the chin downward with moderate force. Then, the patient was instructed to resist the force and hold it for 5-10 seconds. This exercise program was performed twice a day for a

30-minute session in a span of 2 weeks. At the bedside, the patient's guardian frequently conducted passive jaw opening exercise by using two tongue depressors as leverage between upper and lower molar teeth.

Since commonly used evaluation parameters for the TMJ function include MMO and craniomandibular index (CMI), the patient's TMJ functional improvement was assessed with these parameters. The CMI evaluates the extent of TMJ dysfunction and the tenderness severity of the orofacial and neck muscles, and has been found to be reliable for quantifying the severity of temporomandibular disorders ${ }^{11}$. And overall swallowing ability was measured with the Mann Assessment of Swallowing Ability (MASA), which is a valid and reliable clinical assessment tool of dysphagia in a bedside setting ${ }^{12}$. The results showed that two weeks of intensive stomatognathic alignment and TMJ strengthening exercise program significantly improved TMJ function and overall swallowing ability.(Table 1, 2) (Fig. 3) Clinically, the patient was able to chew gum thoroughly in 10 minutes. Also, soft

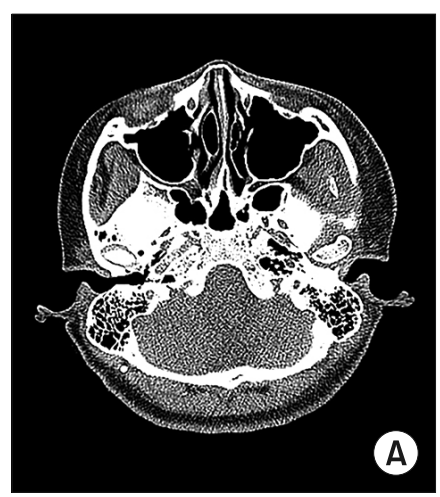

Fig. 2. Facial bone CT of TMJ. (A) Axial view. (B) Coronal view.

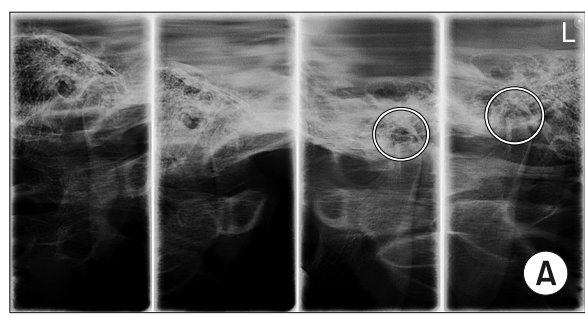

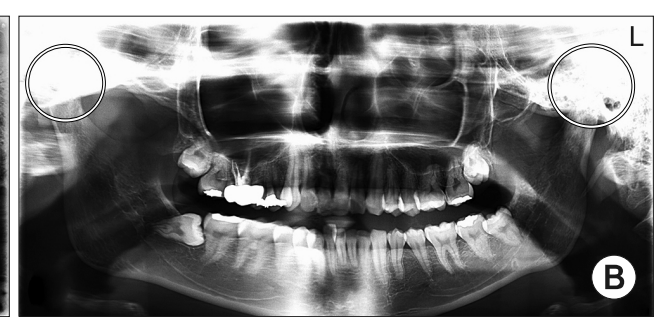

Fig. 1. Radiographic images of TMJ. (A) TMJ Tomogram. (B) Panoramic Dental X-ray. 
Table 1. CMI parameters before and after stomatognathic alignment and TMJ exercises.

\begin{tabular}{|c|c|c|}
\hline \multicolumn{3}{|c|}{ Mandibular Movement (MM) (Normal Values in Parenthesis) } \\
\hline & $\begin{array}{l}\text { Before exercise } \\
(2020-03-16)\end{array}$ & $\begin{array}{c}\text { After exercise } \\
(2020-03-30)\end{array}$ \\
\hline Maximum opening (incisor to incisor) $(40-60 \mathrm{~mm})^{*}$ & $10 \mathrm{~mm}$ & $21 \mathrm{~mm}$ \\
\hline Passive Stretch Opening $(42-62 \mathrm{~mm})^{*}$ & $13 \mathrm{~mm}$ & $27 \mathrm{~mm}$ \\
\hline Restriction on Opening ( $\geq 40 \mathrm{~mm})$ & Positive & Positive \\
\hline Pain on Opening & Negative & Negative \\
\hline Jerky Opening & Negative & Negative \\
\hline "S" Deviation on Opening or Closing ( $\leq 2 \mathrm{~mm})$ & Negative & Negative \\
\hline Lateral Deviation at full Opening & Positive & Positive \\
\hline Protrusion - Pain & Negative & Negative \\
\hline Protrusion - Limitation $(\geq 7 \mathrm{~mm})^{*}$ & $2 \mathrm{~mm}$ & $3 \mathrm{~mm}$ \\
\hline Right Laterotrusion - Pain* & Positive & Negative \\
\hline Right Laterotrusion - Limitation $(\geq 7 \mathrm{~mm})^{*}$ & $1 \mathrm{~mm}$ & $6 \mathrm{~mm}$ \\
\hline Left Laterotrusion - Pain & Positive & Positive \\
\hline Left Laterotrusion - Limitation ( $\geq 7 \mathrm{~mm})$ & $7 \mathrm{~mm}$ & $7 \mathrm{~mm}$ \\
\hline Clinically can lock open (subluxate) & Negative & Negative \\
\hline Clinically can be or is locked closed with no translation of condyle, right or left & Negative & Negative \\
\hline Rigidity of Jaw Upon Manipulation & Positive & Positive \\
\hline \multicolumn{3}{|l|}{ TMJ Noise (TN) } \\
\hline Reciprocal click (Eliminated with Mandibular Repositioning) & Negative & Negative \\
\hline Reproducible Opening Click* & Positive, right & Negative \\
\hline Reproducible Laterotrusive Click Only & Negative & Negative \\
\hline Reproducible Closing Click & Negative & Negative \\
\hline Nonreproducible Opening Click & Negative & Negative \\
\hline Crepitus - Fine & Negative & Negative \\
\hline Crepitus - Coarse & Negative & Negative \\
\hline Popping (audible without stethoscope) & Negative & Negative \\
\hline
\end{tabular}

*Improved CMI parameter.

solid foods such as soups with very soft chunks, ripe fruits like bananas were clinically feasible. After discharge, the patient continued with stomatognathic alignment and TMJ strengthening exercise with the help from the guardian.

By the time the patient was admitted to department of rehabilitation at tertiary hospital for the 4 th time on June 25th, 2020, MMO had been improved from $27 \mathrm{~mm}$ to $33 \mathrm{~mm}$, indicative of an overall improvement of bolus-forming capacity. In comparison to the functional status as of March, 2020, the patient could more readily break down soft solid foods into the form of a bolus.

\section{DISCUSSION}

The stomatognathic system, a functional unit comprised of skeletal components, dental arches, soft tissues, TMJ, and masticatory muscles, act in coordination to perform various functional tasks such as speech, bolus formation, and swallowing ${ }^{13}$. In specific, the TMJ makes muscular and ligamentary connections to the cervical region, forming a functional complex so-called "cranio-cervico-mandibular system." Several studies have shown that patients with TMJD present an excessively forward head position, usually associated with the shortening of the posterior cervical extensor muscles as well as shortening of the $\mathrm{SCM}^{14,15}$. Many of the stroke patients present with impaired head and neck posture and TMJD ${ }^{16}$, and similarly, many of the TBI patients with clear sensorimotor deficits complain of postural instability ${ }^{17}$. Therefore, a great disturbance of the muscular and ligamentary connections of the 
Table 2. MASA parameters before and after stomatognathic alignment and TMJ exercises.

\begin{tabular}{|c|c|c|}
\hline \multicolumn{3}{|c|}{ Mann Assessment of Swallowing Ability (MASA) } \\
\hline & $2020-03-16$ & $2020-03-30$ \\
\hline 1. Alertness & 8=Fluctuating awareness/alert level & 8=Fluctuating awareness/alert level \\
\hline 2. Cooperation & 8=Fluctuating cooperation & 8=Fluctuating cooperation \\
\hline 3. Auditory Comprehension & $\begin{array}{l}6=\text { Follows simple conversation/instructions with } \\
\text { repetition }\end{array}$ & $\begin{array}{l}6=\text { Follows simple conversation/instructions with } \\
\text { repetition }\end{array}$ \\
\hline 4. Respiration & $10=$ Chest clear, no evidence of abnormality & $10=$ Chest clear, no evidence of abnormality \\
\hline 5. Respiratory Rate & $5=$ Able to control breath rate for swallow & $5=$ Able to control breath rate for swallow \\
\hline 6. Dysphasia & $\begin{aligned} 2= & \text { No functional speech, sounds or } \\
& \text { undecipherable singe words }\end{aligned}$ & $\begin{aligned} 2=\text { No functional speech, sounds or } \\
\text { undecipherable singe words }\end{aligned}$ \\
\hline 7. Dyspraxia & $1=$ Unable to assess & $1=$ Unable to assess \\
\hline 8. Dysarthria & $1=$ Unable to assess & $1=$ Unable to assess \\
\hline 9. Saliva* & 2=Some drool consistency & $\begin{array}{l}\text { 3=Drooling at times, during speech while side } \\
\text { lying, when fatigued }\end{array}$ \\
\hline 10. Lip Seal* & $2=$ Incomplete closure, limited movement & $\begin{array}{l}3=\text { Unilateral weak, poor maintenance, restricted } \\
\text { movement }\end{array}$ \\
\hline 11. Tongue Movement & 6=Incomplete movement & 6=Incomplete movement \\
\hline 12. Tongue Strength & 8=Minimal weakness & 8=Minimal weakness \\
\hline 13. Tongue Coordination* & $5=$ Gross incoordination & 8=Mild incoordination \\
\hline 14. Oral Preparation* & $\begin{array}{l}6=\text { Minimal chew/tongue thrust bolus projected } \\
\text { forward }\end{array}$ & $8=$ Lip or tongue seal, bolus escape \\
\hline 15. Gag & 4=Diminished bilaterally & 4=Diminished bilaterally \\
\hline 16. Palate & $8=$ Slight asymmetry noted, mobile & $8=$ Slight asymmetry noted, mobile \\
\hline 17. Bolus Clearance* & $5=$ Some clearance, residue & $8=$ Significant clearance, minimal residue \\
\hline 18. Oral Transit & 4=Delay Greater than 10 seconds & $4=$ Delay Greater than 10 seconds \\
\hline 19. Cough Reflex & 3=Weak reflexive cough & 3=Weak reflexive cough \\
\hline 20. Voluntary Cough & $2=$ No attempt/unable to assess & $2=\mathrm{No}$ attempt/unable to assess \\
\hline 21. Voice & 6=Hoarse, difficulty with pitch/volume control & 6=Hoarse, difficulty with pitch/volume control \\
\hline 22. Trache & $10=$ No trache required & $10=$ No trache required \\
\hline 23. Pharyngeal Phase & $\begin{array}{c}5=\text { Incomplete laryngeal elevation, jerking } \\
\text { progression, pooling on phonation }\end{array}$ & $\begin{array}{c}5=\text { =Incomplete laryngeal elevation, jerking } \\
\text { progression, pooling on phonation }\end{array}$ \\
\hline 24. Pharyngeal Response & 1=Not coping, gurgling & 1=Not coping, gurgling \\
\hline Total & 118 & 128 \\
\hline
\end{tabular}

*Improved MASA parameter.
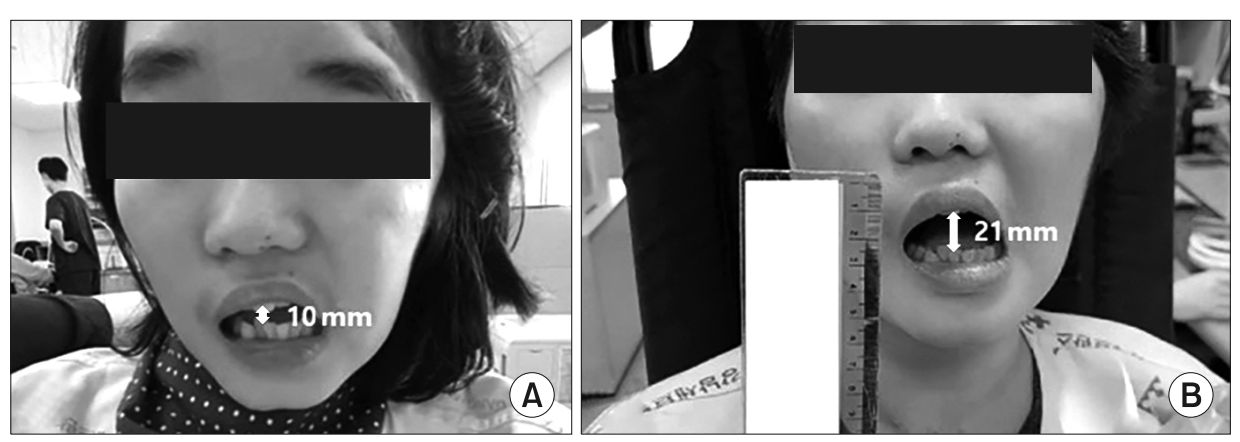

Fig. 3. Maximal mouth opening. (A) Before exercise program. (B) After exercise program. stomatognathic system could be expected in acquired brain injury patients. With regard to this perspective on the pathophysiological perspective of TMJD, we hypothesized that stomatognathic alignment exercise may be able to improve postural alignment of the head and neck, thereby achieving functional improvement of the stomatognathic system. Indeed, a recent study led by $\mathrm{Oh}$ et al. and Volkan et al. 
concluded that stomatognathic alignment exercise improves the quality of swallowing function in stroke patients with limited mouth opening, ${ }^{9,10}$.

In this case study, stomatognathic alignment and TMJ strengthening exercise program over the span of two weeks was applied to the patient, and then the therapeutic effects were evaluated with the CMI and the MASA parameters. After the exercise program was implemented, the CMI parameters such as MMO, protrusion, and Rt. laterotrusion showed improvement, suggestive of a greater range of the mandibular movement.(Table 1) Meanwhile, MASA score improved from 118 to 128, in which a higher score indicates better swallowing function. The improvements were made primarily in the oral phase parameters such as secretions management, lip seal, tongue coordination, oral preparation, and bolus clearance. These findings indicate that postural adjustment and augmentation of TMJ muscles can be effective in improvement of neck mobility and orofacial movements. It is worth pointing out that although diet level 6 (soft and bite-sized food) was tolerable to the patient according to the previous VFSS results, the patient still could not efficiently eat soft and bite-sized foods due to poor oral preparatory and oral phase prior to the implementation of our designed exercise program. However, after only two weeks of the exercise program, oral process of bolus formation showed a definite improvement. Afterwards, the patient actually began soft and bite-sized diet in clinical setting. A number of studies have been conducted on the efficacy of stomatognathic alignment exercise program on stroke-related TMJD, yet its effect on TBI-related TMJD has not been mentioned before. Our conclusion shows that active exercise to achieve postural correction and TMJ strengthening could be an effective treatment option for dysphagia associated with TMJD secondary to TBI.

This case study had certain limitations. Because of soft, fluctuating characteristics of 3D printing implant material, the extent to which the patient injured the temporalis muscle was not taken into an accurate measurement with ultrasound. In addition, larger numbers of patients are needed to confirm the efficacy of stomatognathic alignment exercise and TMJ strengthening exercise in brain injury patients with TMJD. Alberto et al. also declared that although therapeutic exercises are considered effective in the management of muscular TMD, the development of randomized clinical trials is necessary since many existing studies are still based on the clinical experience of professionals ${ }^{18}$.

Nevertheless, it is important to invent strategic treatment methods to improve a patient's total dietary intake rather than continuing with conventional dysphagia therapy in a chronic brain injury inpatient.

\section{CONFLICT OF INTEREST}

The authors declare that they have no conflict of interest.

\section{REFERENCES}

1. Shiffman MA. History of cosmetic surgery. Cosmetic Surgery. Springer, 2013:3-28.

2. Anderson GC, Gonzalez YM, Ohrbach R, Truelove EL, Sommers E, Look JO, et al. The Research Diagnostic Criteria for Temporomandibular Disorders. VI: future directions. J Orofac Pain. 2010;24:79-88.

3. Moore K, Dalley A. II, Agur AM: Clinically Oriented Anatomy. Lippincott Williams \& Wilkins, New York, 2017.

4. Horan F. Gray's Anatomy: the anatomical basis of clinical practice: Edited by Susan Standring Pp. 1551. Illinois: Churchill Livingstone Elsevier, 2008. ISBN: 9780-443-06684-9.£ 99. The British Editorial Society of Bone and Joint Surgery, 2009.

5. Wright EF, Klasser GD. Manual of temporomandibular disorders. John Wiley \& Sons, 2019.

6. Lam P, Stanschus S, Zaman R, Cichero JA. The international dysphagia diet standardisation initiative (IDDSI) framework: The kempen pilot. British Journal of Neuroscience Nursing. 2017;13:S18-S26.

7. Schimmel M, Ono T, Lam OL, Müller F. Oro-facial impairment in stroke patients. J Oral Rehabil. 2017;44: 313-26.

8. Newlands C, Kerawala C. Oral and maxillofacial surgery. Oxford University Press, 2020.

9. Oh DW, Kang TW, Kim SJ. Effect of stomatognathic alignment exercise on temporomandibular joint function and swallowing function of stroke patients with limited 
mouth opening. J Phys Ther Sci. 2013;25:1325-9.

10. Yilmaz V, Aras B, Umay E. Temporomandibular Joint Dysfunction and Impaired Stomatognathic Alignment: A Problem Beyond Swallowing in Patients With Stroke. Indian J Otolaryngol Head Neck Surg. 2020;72:329-34.

11. Fricton JR, Schiffman EL. The craniomandibular index: validity. The Journal of prosthetic dentistry. 1987;58: 222-8.

12. Karaca Umay E, Gündoğdu İ, Gürçay E, Öztürk E, Yılmaz $\mathrm{V}$, Karaahmet Ö, et al. The psychometric evaluation of the Turkish version of the Mann Assessment of Swallowing Ability in patients in the early period after stroke. Turk J Med Sci. 2018;48:1153-61.

13. Cuccia A, Caradonna C. The relationship between the stomatognathic system and body posture. Clinics (Sao Paulo). 2009;64:61-6.

14. Hackney J, Bade D, Clawson A. Relationship between forward head posture and diagnosed internal derangement of the temporomandibular joint. J Orofac Pain. 1993;7:386-90.

15. Olmos SR, Kritz-Silverstein D, Halligan W, Silverstein ST. The effect of condyle fossa relationships on head posture. Cranio. 2005;23:48-52.

16. Borello-France DF, Burdett RG, Gee ZL. Modification of sitting posture of patients with hemiplegia using seat boards and backboards. Phys Ther. 1988;68:67-71.

17. Geurts AC, Ribbers GM, Knoop JA, van Limbeek J. Identification of static and dynamic postural instability following traumatic brain injury. Arch Phys Med Rehabil. 1996;77:639-44.

18. Moraes Ada R, Sanches ML, Ribeiro EC, Guimarães AS. Therapeutic exercises for the control of temporomandibular disorders. Dental Press J Orthod. 2013;18:134-9. 